\title{
Radiation transport in diffractive media
}

\author{
Mattias Marklund \\ Department of Physics, Umeå University, SE-901 87 Umeå, Sweden
}

\begin{abstract}
We consider radiation transport theory applied to non-dispersive but refractive media. This setting is used to discuss Minkowski's and Abraham's electromagnetic momentum, and to derive conservation equations independent of the choice of momentum definition. Using general relativistic kinetic theory, we derive and discuss a radiation gas energy-momentum conservation equation valid in arbitrary curved spacetime with diffractive media.
\end{abstract}

PACS numbers: 41.20.Jb, 05.60.-k, 42.15.-i

\section{Introduction}

Radiative transfer is a mature area of research, with important application both in laboratory and astrophysical systems (see, e.g., $[1,2]$ and references therein). Much is thus known about the properties of the relevant radiation transport equations in non-dispersive, non-diffractive media in flat spacetime. However, less is known in the case of dispersive and/or diffractive media, and the addition of spacetime curvature reduces the number of relevant publications further (for a representative but incomplete selection see [2-8]). Different ways to tackle the problems involved in formulating a general theory of curved spacetime radiation transport in diffractive media exists in the literature. In many cases, the treatment of moments of the transfer equations, and their respective conservation equation, is lacking, as is the discussion of the interpretation of the radiation fluid moments. The latter can be viewed as a nontrivial task, since a clear-cut definition of the radiation momentum density is still under discussion (although claimed otherwise by some authors, see, e.g., [9]). There are exceptions in the literature though. For example, Anderson \& Spiegel introduces an optical geometry on top of the curved spacetime, by introducing an effective metric which incorporates the refractive index of the medium (similar to Gordon [10]). In this effective geometry, the transfer equation takes the standard form, and a treatment of the fluid moments is thus straightforward, although their interpretation is somewhat obscured by the presence of the effective geometry. Moreover, the proper conservation equations for the fluid moments is not derived.

As noted by many authors, related to the problem of radiative transfer in diffractive media is concept of photon momentum and the Minkowski-Abraham debate. Although the definition of Abraham is preferred due to a number of theoretical reasons 
(symmetries [9, 11], derivations from microscopic theory [12] etc., see [13] for an overview), measurements have not given a definite answer [14,15] (for a review, see [16] and references therein). Due to the problem of separating the electromagnetic from the material degrees of freedom $[17,18]$, the problem of defining the electromagnetic momentum in a refractive medium has persisted. However, it seems reasonable that the Minkowski momentum should be treated as a pseudo-momentum, partly depending on material contributions, while the Abraham momentum is a proper electromagnetic momentum (for a discussion, see [19]).

Here we will analyse the radiative transfer equations and derive macroscopic conservation equations in terms of both Abraham's and Minkowski's definitions. It will also be shown that variables can be used as to remove the problem of momentum definition when deriving conservation equations. Furthermore, a completely general energy-momentum conservation equation for a radiation fluid in curved spacetime with a refractive medium is derived and discussed. It is shown that the combination of curvature and refraction gives important contributions to the conservation equation, even if the refractive index is spacetime homogeneous. The equation is discussed in the context of cosmological models.

\section{Ray dynamics and kinetic theory}

In mechanics, given the action $S$ of a system, we may define the momentum and Hamiltonian according to

$$
p_{\alpha}=\frac{\partial S}{\partial x^{\alpha}} \quad H=-\frac{\partial S}{\partial t}
$$

respectively. From this, the equations of motion for a single particle can be written in terms of Hamilton's equations, i.e.

$$
\begin{aligned}
& \dot{x}^{\alpha}=\frac{\partial H}{\partial p_{\alpha}}, \\
& \dot{p}_{\alpha}=-\frac{\partial H}{\partial x^{\alpha}} .
\end{aligned}
$$

In geometric optics [11], we introduce the eikonal $\phi=\phi\left(t, x^{\alpha}\right)$ by writing the electromagnetic field $F$ in the form

$$
F=a \exp (i \phi)
$$

From the eikonal, we define the the wave-vector and frequency of the field as

$$
k_{\alpha}=\frac{\partial \phi}{\partial x^{\alpha}} \quad \omega=-\frac{\partial \phi}{\partial t},
$$

in analogy with (11). The equations of ray optics becomes

$$
\begin{aligned}
& \dot{x}^{\alpha}=\frac{\partial \omega}{\partial k_{\alpha}}, \\
& \dot{k}_{\alpha}=-\frac{\partial \omega}{\partial x^{\alpha}},
\end{aligned}
$$


and from this also follows that $d \omega / d t=\partial \omega / \partial t$. These equations are the mass-less quasi-particle analogous of (21). It is also clear from the eikonal definitions that the natural variables describing the photons are the frequency and the wave-number rather than the energy and the momentum.

Photons moving in a isotropic non-dispersive but refractive medium will satisfy a dispersion relation of the form

$$
\omega=\frac{c k}{n}
$$

where, in general, the refractive index $n$ is spacetime inhomogeneous. The equations of motion (2) for individual photons in such a medium then becomes

$$
\begin{aligned}
\dot{x}^{\alpha} & =\frac{c}{n} \hat{k}^{\alpha}, \\
\dot{k}_{\alpha} & =\frac{\omega}{n} \frac{\partial n}{\partial x^{\alpha}} .
\end{aligned}
$$

Before proceeding further, a discussion of the definition of the photon momentum is in place. We note that we have chosen as our fundamental variables $t, x^{\alpha}$ and $\omega, k_{\alpha}$. The relation to Hamiltonian particle dynamics becomes obvious if we choose $H=\hbar \omega$ and $p_{\alpha}=\hbar k_{\alpha}$, and it is therefore tempting to assume this direct relationship. However, in electromagnetic theory, there are two distinct ways to define the momentum of the electromagnetic field. According to Minkowski, the momentum is proportional to $\mathbf{D} \times \mathbf{B}$, while according to Abraham it is proportional to $\mathbf{E} \times \mathbf{H}$ (see Ref. [9] for a discussion). Furthermore, in measurement of photon momenta in isotropic diffractive media, there are two distinct forms as well, namely $p=\hbar k=\hbar \omega n / c$ and $p \equiv \hbar \omega / n c=\hbar k / n^{2}$. We note that the former is in agreement with Minkowski's momentum density and a number of experiments, while the latter definition is consistent with Abraham's choice in terms of the Poynting flux (see [16] and references therein), which also follows from detailed microscopic considerations as well as symmetry arguments. On the other hand, measurements indicates that $p \propto n$, consistent with Minkowski's momentum definition in a dielectric medium. The discrepancy between the two definitions can be attributed to the contributions of the medium in Minkowski's definition, while Abraham's definition (used here) is the 'proper' momentum of the photon [19]. However, we note that while the form (17a) of the group velocity is valid independently of the momentum definition (as expected), (7b) formulated in terms of the momentum takes different forms for the Minkowski and the Abraham definition.

Given a spectral distribution $\mathscr{N}\left(t, x^{\alpha}, k_{\alpha}\right)$ of photons, the absence of collisions defines the on-shell Vlasov equation for $\mathscr{N}$ according to

$$
\dot{\mathscr{N}}=\frac{\partial \mathscr{N}}{\partial t}+\dot{x}^{\alpha} \frac{\partial \mathscr{N}}{\partial x^{\alpha}}+\dot{k}_{\alpha} \frac{\partial \mathscr{N}}{\partial k_{\alpha}}=0
$$

expressing the phase space conservation of quasi-particles.

Using the spectral distribution function defined on-shell, i.e. with (6) satisfied, we can now define the macroscopic observables, from which a fluid theory can be constructed. 


\section{Macroscopic variables}

Next, we define macroscopic variables as moments of the distribution function $\mathscr{N}$. In general, we define $\langle\psi\rangle=\left(\int \mathscr{N} d^{3} k\right)^{-1} \int \psi \mathscr{N} d^{3} k$ to be the statistical average of the function $\psi$, which may be defined over the full phase space. Moreover, in these definitions we closely follow Ref. [1].

- The number density $N$ is defined according to

$$
N=\int \mathscr{N} d^{3} k
$$

- The energy density $\mu$ is defined as

$$
\mu=\int \hbar \omega \mathscr{N} d^{3} k
$$

- The average fluid velocity $u^{\alpha}$ is given by

$$
u^{\alpha}=\left\langle\dot{x}^{\alpha}\right\rangle
$$

and from this we may also define the thermal (or random) fluid velocity $w^{\alpha}$ using

$$
w^{\alpha}=\dot{x}^{\alpha}-u^{\alpha},
$$

such that $\left\langle w^{\alpha}\right\rangle=0$.

- The radiation energy flux $q^{\alpha}$ is defined such that $q^{\alpha} d S_{\alpha}$ is the rate of energy flow across the surface $d S_{\alpha}$. Thus

$$
q^{\alpha}=\int \hbar \omega w^{\alpha} \mathscr{N} d^{3} k
$$

- The momentum density $\Pi^{\alpha}$ of the photon in a refractive medium can be formulated in two ways, in accordance with Minkowski or Abraham, and they read

$$
\Pi_{M}^{\alpha}=\int \hbar k^{\alpha} \mathscr{N} d^{3} k=\int \frac{n^{2}}{c^{2}} \hbar \omega \dot{x}^{\alpha} \mathscr{N} d^{3} k,
$$

and

$$
\Pi_{A}^{\alpha}=\int \frac{\hbar k^{\alpha}}{n^{2}} \mathscr{N} d^{3} k=\int \frac{1}{c^{2}} \hbar \omega \dot{x}^{\alpha} \mathscr{N} d^{3} k
$$

respectively, where we have made use of (6) $-(7 b)$.

- The pressure tensor $\mathrm{P}^{\alpha \beta}$ can similarly be defined in two distinct ways. Since the pressure tensor $\mathrm{P}^{\alpha \beta}$ is defined to be, for an observer comoving with the average fluid flow, the rate of transport of the $\alpha$ component of momentum per unit area of a surface orthogonal to the $\beta$ coordinate (or tetrad) axis, we have

$$
\mathrm{P}_{M}^{\alpha \beta}=\int \hbar k^{\alpha} w^{\beta} \mathscr{N} d^{3} k=\int \frac{n^{2}}{c^{2}} \hbar \omega w^{\alpha} w^{\beta} \mathscr{N} d^{3} k
$$

and

$$
\mathrm{P}_{A}^{\alpha \beta}=\int \frac{1}{n^{2}} \hbar k^{\alpha} w^{\beta} \mathscr{N} d^{3} k=\int \frac{1}{c^{2}} \hbar \omega w^{\alpha} w^{\beta} \mathscr{N} d^{3} k
$$

for the Minkowski and the Abraham momentum, respectively. Here we have again used (6) $-(7 b)$, and we note that the pressure tensor is symmetric. 
Furthermore, the energy flux and the momentum density is related to each other through the relations

$$
\Pi_{M}^{\alpha}=\frac{n^{2}}{c^{2}}\left(\mu u^{\alpha}+q^{\alpha}\right)
$$

and

$$
\Pi_{A}^{\alpha}=\frac{1}{c^{2}}\left(\mu u^{\alpha}+q^{\alpha}\right),
$$

where we have used general relation $k^{\alpha}=n^{2} \omega\left(u^{\alpha}+w^{\alpha}\right) / c^{2}$.

Higher order moments can be defined analogously, but the above will suffice for our purposes, and with these definitions we are ready to set up the fluid equations. We note that the macroscopic quantities defined above are valid also for dispersive media, i.e. when the refractive index depend on the frequency $\omega$ or, in the anisotropic case, the wave vector $k^{\alpha}$.

\section{Fluid equations}

A hierarchy of fluid equations may be obtained from (8) by taking the moments with respect to suitable microscopic quantities. Given a microscopic variable $\psi$ (which may be a tensorial object), the general fluid conservation equation takes the form

$$
\frac{\partial}{\partial t}(N\langle\psi\rangle)+\frac{\partial}{\partial x^{\alpha}}\left(N\left\langle\dot{x}^{\alpha} \psi\right\rangle\right)=N\langle\dot{\psi}\rangle
$$

where $\dot{\psi}$ is defined in accordance with (8). Thus, $N\langle\psi\rangle$ and $N\left\langle\dot{x}^{a} \psi\right\rangle$ represent the macroscopic density and macroscopic current, respectively, of the microscopic variable $\psi$, while the right hand side of (17) acts as a source/sink. Finally, the fluid hierarchy of equation can be terminated by assuming a set of thermodynamic relationships between the macroscopic variables, such as an equation of state.

Putting $\psi=1$, we obtain the conservation equation for the number of quasiparticles

$$
\frac{\partial N}{\partial t}+\frac{\partial}{\partial x^{\beta}}\left(N u^{\beta}\right)=0
$$

The energy conservation equation is obtained by taking $\psi=\hbar \omega$. Thus, for an observer comoving with the average radiation fluid flow we obtain

$$
\frac{\partial \mu}{\partial t}+\frac{\partial}{\partial x^{\alpha}}\left(\mu u^{\alpha}+q^{\alpha}\right)=-\frac{\mu}{n} \frac{\partial n}{\partial t} .
$$

Similarly, the momentum conservation equation is obtained by $\psi=p_{\alpha}$, and takes the form

$$
\frac{\partial \Pi_{M \alpha}}{\partial t}+\frac{\partial}{\partial x^{\beta}}\left(u^{\beta} \Pi_{M \alpha}+\frac{n^{2}}{c^{2}} u_{\alpha} q^{\beta}+\mathrm{P}_{M \alpha}^{\beta}\right)=\frac{\mu}{n} \frac{\partial n}{\partial x^{\alpha}}
$$

or

$$
\begin{aligned}
\frac{\partial \Pi_{A \alpha}}{\partial t}+\frac{\partial}{\partial x^{\beta}}\left(u^{\beta} \Pi_{A \alpha}+\frac{1}{c^{2}} u_{\alpha} q^{\beta}+\mathrm{P}_{A \alpha}^{\beta}\right)= & \frac{\mu}{n^{3}} \frac{\partial n}{\partial x^{\alpha}}-2 \frac{\Pi_{A \alpha}}{n}\left(\frac{\partial}{\partial t}+u^{\beta} \frac{\partial}{\partial x^{\beta}}\right) n \\
& -\frac{2}{n}\left(\frac{1}{c^{2}} u_{\alpha} q^{\beta}+\mathrm{P}_{A \alpha}^{\beta}\right) \frac{\partial n}{\partial x^{\beta}},
\end{aligned}
$$


depending on if we use (14a) and (15a) or (14b) and (15b), respectively. We furthermore note that $(20 a)$ and $(20 b)$ can be written in a slightly more symmetric form, using the relations (16a)-(16b), according to

$$
\frac{\partial \Pi_{M}^{\alpha}}{\partial t}+\frac{\partial}{\partial x^{\beta}}\left(\frac{n^{2}}{c^{2}} \mu u^{\alpha} u^{\beta}+\frac{2 n^{2}}{c^{2}} u^{(\alpha} q^{\beta)}+\mathrm{P}_{M}^{\alpha \beta}\right)=\frac{\mu}{n} \delta^{\alpha \beta} \frac{\partial n}{\partial x^{\beta}}
$$

and

$$
\begin{aligned}
\frac{\partial \Pi_{A}^{\alpha}}{\partial t}+\frac{\partial}{\partial x^{\beta}}\left(\frac{1}{c^{2}} \mu u^{\alpha} u^{\beta}+\frac{2}{c^{2}} u^{(\alpha} q^{\beta)}\right. & \left.+\mathrm{P}_{A}^{\alpha \beta}\right)=\frac{\mu}{n^{3}} \delta^{\alpha \beta} \frac{\partial n}{\partial x^{\beta}}-\frac{2}{n} \Pi_{A}^{\alpha} \frac{\partial n}{\partial t} \\
- & \frac{2}{n}\left(\frac{1}{c^{2}} \mu u^{\alpha} u^{\beta}+\frac{2}{c^{2}} u^{(\alpha} q^{\beta)}+\mathrm{P}_{A}^{\alpha \beta}\right) \frac{\partial n}{\partial x^{\beta}}
\end{aligned}
$$

respectively. It is straighforward to see that $(20 a)-(20 b)$ can be obtained from each other using the relation $\Pi_{M}^{\alpha}=n^{2} \Pi_{A}^{\alpha}$.

The system of equations presented above can be closed if we choose a thermodynamic relationship between certain quantities. For an close-to-equilibrium system, the pressure tensor becomes nearly isotropic, and we can write $\mathrm{P}^{\alpha \beta} \approx P h^{\alpha \beta}$, where $P=h_{\alpha \beta} \mathrm{P}^{\alpha \beta} / 3$. From (15a) and (15b), we obtain

$$
P_{M}=\frac{1}{3} \mu \quad P_{A}=\frac{1}{3} n^{-2} \mu,
$$

respectively.

The remaining freedom in the equations is removed by choosing the observer. We will here use the particle frame, where $u^{\alpha}=0$.

Using these choices, we obtain a set of equations independent of the choice of momentum definition, in terms of the energy density and heat flux, according to

$$
\frac{\partial \mu}{\partial t}+\frac{\partial q^{\alpha}}{\partial x^{\alpha}}=-\frac{\mu}{n} \frac{\partial n}{\partial t}
$$

and

$$
\frac{\partial q^{\alpha}}{\partial t}+\frac{c^{2}}{3 n^{2}} \delta^{\alpha \beta} \frac{\partial \mu}{\partial x^{\beta}}=\frac{c^{2} \mu}{n^{3}} h^{\alpha \beta} \frac{\partial n}{\partial x^{\beta}}-\frac{q^{\alpha}}{n} \frac{\partial n}{\partial t},
$$

from (16a $)$ - (16b), (19), and (20a $)-(20 b)$, respectively. We may from this derive general wave equations for the energy density and energy flux for radiation in diffractive media.

\section{Covariant conservation laws}

In this section we will make use of the $1+3$ orthonormal frame (ONF) approach (see [20] for an overview), since it allows for a general relativistic treatment, simplifies calculations, and gives the natural Cartesian like reference frame for an observer moving with the timelike frame direction. All vector and tensor quantities will be projected onto this frame.

We define a set of frame vectors $\left\{\boldsymbol{e}_{a}\right\}, a=0, \ldots, 3$ such that $\boldsymbol{e}_{a} \cdot \boldsymbol{e}_{b}=\eta_{a b}$ gives the constant coefficients of the metric: $g_{a b}=\eta_{a b}=\operatorname{diag}(-1,1,1,1)$, i.e., a Lorentz frame. The Ricci rotation coefficients $\Gamma_{b c}^{a}$, antisymmetric in the first two indices, giving 
the kinematics of spacetime are defined by $\nabla_{c} \boldsymbol{e}_{b}=\Gamma_{b c}^{a} \boldsymbol{e}_{a}$. Here $\nabla_{c}$ is the covariant derivative with respect to the Lorentz frame.

The distribution function $\tilde{\mathscr{N}}$ will now be a function of the canonical phase space variables $x^{a}$ and $k_{a}$, and the conservation of phase space density can be written

$$
\frac{d \tilde{\mathscr{N}}}{d \lambda}=\hat{L}[\tilde{\mathscr{N}}]=\dot{x}^{a} \boldsymbol{e}_{a}(\tilde{\mathscr{N}})+\dot{k}_{a} \frac{\partial \tilde{\mathscr{N}}}{\partial k_{a}}=\mathscr{C}
$$

where the over dot stands for $d / d \lambda$, where $\lambda$ is an affine parameter along the photon path, and we have introduced the Liouville operator $\hat{L}=\dot{x}^{a} \boldsymbol{e}_{a}+\dot{k}_{a} \partial / \partial k_{a}$. Furthermore, for the sake of generality, we have added the collisional operator $\mathscr{C}$, representing photon emission and absorption $\ddagger$. If $f^{a}$ are the external forces acting on the pencil of light, the covariant derivative along the photon path is

$$
\frac{D k_{b}}{d \lambda}=\dot{k}_{b}-\Gamma_{b a}^{c} k_{c} \dot{x}^{a}=f_{b}
$$

With the Hamiltonian $H=H\left(x^{a}, k_{a}\right)$ in eight dimensional phase space, the equations of motion thus reads

$$
\begin{aligned}
\dot{x}^{a} & =\frac{\partial H}{\partial k_{a}}, \\
\dot{k}_{a} & =-\boldsymbol{e}_{a}(H)+\Gamma_{a b}^{c} k_{c} \dot{x}^{b},
\end{aligned}
$$

generalising ( $(5 a)-(5 b)$. Here the last term of $(27 b)$ gives the gravitational contribution to the equations of motion. We denote the observer four-velocity by $U^{a}$, normalised such that $U^{a} U_{a}=-c^{2}$. We partially fix the frame by letting the observer four-velocity $U^{a}=\delta_{0}^{a}$, and split spacetime quantities with respect to $U^{a}$. The space metric orthogonal to $U^{a}$ takes the form

$$
h_{a b}=\eta_{a b}+U_{a} U_{b} / c^{2}
$$

and the wavevector can be written

$$
k_{a}=\omega U_{a} / c^{2}+k \ell_{a},
$$

where $\omega=-U^{a} k_{a}, k=\left(h^{a b} k_{a} k_{b}\right)^{1 / 2}$, and $\ell_{a}=h_{a}^{b} k_{b} / k$. We note that $\ell^{a} \ell_{a}=1$ and $\ell_{a} U^{a}=0$. In these variables, the dispersion relation (6) retains its form.

There is a certain arbitrariness in the choice of Hamiltonian. We choose the Hamiltonian $H$ such that $H=0$ gives the dispersion relation (and $\partial H / \partial k_{a} \neq 0$ on the dispersion surface), and the variables $x^{a}, \omega, k$, and $\ell_{a}$ is treated as independent, with the vanishing Hamiltonian as a constraint. Suppose the dispersion relation takes the form

$$
\omega=W\left(x^{a}, k, \ell_{a}\right) .
$$

$\ddagger$ The process of emission and absorption is thoroughly treated in any book on radiation transport, see e.g. [1], and will therefore not be discussed further here, apart from the following brief comment. As an example, in case of applications to the early universe, the major contribution to the collisional term would be in the form of Thomson scattering, but there are of course numerous other scattering events that could dominate in other parameter regimes. 
Then

$$
H=-\omega+W\left(x^{a}, k, \ell_{a}\right)
$$

satisfies the necessary criteria listed above, giving the equations of motion

$$
\begin{aligned}
& \dot{x}^{a}=U^{a}+\frac{\partial W}{\partial k_{a}}, \\
& \dot{k}_{a}=-\boldsymbol{e}_{a}(W)+\Gamma_{a b}^{c} k_{c} \dot{x}^{b},
\end{aligned}
$$

via (27a) $-(27 b)$, and, moreover, $\dot{H}=e_{0}(H)=e_{0}(W)$. With (6) $), W\left(x^{a}, k\right)=k c / n\left(x^{a}\right)$, and

$$
\begin{aligned}
& \frac{\partial W}{\partial k_{a}}=\frac{c \ell^{a}}{n}, \\
& \boldsymbol{e}_{a}(W)=-\frac{\omega}{n} \boldsymbol{e}_{a}(n) .
\end{aligned}
$$

Thus, with the particular dispersion relation (6), the kinetic equation (25) becomes

$$
\left(U^{a}+\frac{c \ell^{a}}{n}\right) \boldsymbol{e}_{a}(\tilde{\mathscr{N}})+\left[\frac{\omega}{n} \boldsymbol{e}_{a}(n)+\Gamma_{a b}^{c} k_{c} \dot{x}^{b}\right] \frac{\partial \tilde{\mathscr{N}}}{\partial k_{a}}=\mathscr{C} .
$$

In the literature, the common definition of the energy-momentum tensor is

$$
T_{M}^{a b}=\hbar \int k^{a} k^{b} \tilde{\mathscr{N}} \boldsymbol{\kappa}
$$

where $\boldsymbol{\kappa}=|\operatorname{det} g|^{-1 / 2}(\delta(H) / \omega) d^{4} k$ is the invariant volume measure in momentum space. This definition is consistent with the Minkowski definition of the photon momentum in diffractive media. Furthermore, in non-diffractive media, this is a conserved quantity, due to the one-to-one correspondence between $\dot{x}^{a}$ and $k_{a}$. However, in diffractive media, this correspondence is lost, since

$$
\dot{x}^{a}=c^{2} \frac{k^{a}}{\omega}+\frac{c}{n}\left(1-n^{2}\right) \ell^{a}=\frac{c^{2}}{\omega}\left[g^{a b}-\left(1-\frac{1}{n^{2}}\right) h^{a b}\right] k_{b}
$$

[see $(\underline{32 a})$ and $(\underline{33 a})]$. Thus, in diffractive media, the energy-momentum tensor may alternatively be defined according to

$$
T_{A}^{a b}=\hbar \int \frac{\omega^{2}}{c^{2}} \frac{\dot{x}^{a}}{c} \frac{\dot{x}^{b}}{c} \tilde{\mathscr{N}} \boldsymbol{\kappa}=\frac{\hbar}{c^{4}} \int \omega \dot{x}^{a} \dot{x}^{b} \tilde{\mathscr{N}} \delta(H) d^{4} k,
$$

in accordance with Abraham's photon momentum definition. We note that when $n \rightarrow 1$, we regain the common definition of the energy-momentum tensor in non-diffractive media. Moreover, since (36) holds in general, we have the relation

$$
T_{A}^{a b}=\left[g_{c}^{a}-\left(1-\frac{1}{n^{2}}\right) h_{c}^{a}\right]\left[g_{d}^{b}-\left(1-\frac{1}{n^{2}}\right) h_{d}^{b}\right] T_{M}^{c d}
$$

between the two definitions of the energy-momentum definition.

From any energy-momentum tensor, we may define the fluid quantities used in the preceding sections. We thus have the relativistic energy density $\mu=T_{a b} U^{a} U^{b}$ with respect to $U^{a}$, the relativistic momentum density $\Pi^{a}=-h^{a b} T_{b c} U^{c}$, the scalar pressure $P=\left(c^{2} T_{a b} h^{a b}\right) / 3$, and the full anisotropic pressure $\mathrm{P}_{a b}=T_{c d} h_{a}^{c} h_{b}^{d}$. By construction $\Pi^{a}$ and $\mathrm{P}_{a b}$ are spacelike quantities, i.e., orthogonal to $U^{a}$. 
The equations of conservation of energy and momentum can be obtained by multiplying (34) by $\hbar \omega^{2} \dot{x}^{b} / c^{4}$, and integrating over the momentum space variables on the dispersion surface. The result is the energy-momentum conservation equation

$$
\begin{gathered}
\nabla_{a} T_{A}^{a b}=2\left(1-\frac{1}{n^{2}}\right) \Gamma_{a d}^{c}\left[n^{2} g^{a(b} h_{c e}-h^{a(b} g_{c e}-n^{2}\left(1-\frac{1}{n^{2}}\right) h^{a(b} h_{c e}\right] T_{A}^{d) e} \\
+\frac{1}{c^{2}}\left\{\left[g^{a b}-\left(1-\frac{1}{n^{2}}\right) h^{a b}\right] U_{d} U_{e}+2 U^{a} U_{d} g_{e}^{b}-4 h^{(a}{ }_{d} g^{b)}{ }_{e}\right\} T_{A}^{d e} \frac{\nabla_{a} n}{n}+C^{b},
\end{gathered}
$$

where $C^{b}=\int\left(\hbar \omega^{2} \dot{x}^{b} / c^{4}\right) \mathscr{C} \kappa$ is the collisional contribution, and $\nabla_{a}$ is the covariant derivative with respect to the Lorentz frame. We note that as $n \rightarrow 1$, all terms on the right hand side, except $C^{b}$, vanishes and we obtain the standard conservation equation for non-diffractive media. Moreover, using the transformation (38) we obtain the corresponding equation in terms of the Minkowski variables. The first term on the right hand side gives the coupling between spacetime kinematics and the media refractive properties. Thus, when we have either flat spacetime or non-diffractive media, this term vanishes. The second term arises from the spatial and/or time-like dependence of the refractive index. Equation (39) gives the evolution of energy and momentum of a radiation fluid in diffractive media on an arbitrary curved spacetime. Thus, (39) is the proper starting point for analysing photon gas dynamics in general relativistic gravity. As compared to the case of a Minkowski background spacetime, there is a significant alteration to the equation due to the novel coupling between gravity and the diffractive media, as given by the first term on the right hand side of (39).

As a simple example of curvature effects, we use (39) to derive the appropriate energy conservation equation in a Friedmann-Lemaitre-Robertson-Walker spacetime, i.e. spatial homogeneity and isotropy is assumed. The spacetime is characterised by the time dependent scalar quantities $\mu$ (energy density), $P$ (pressure), $\Theta$ (expansion), and $n$ (refractive index) (see [20] for details). Thus, (39) gives

$$
\frac{d \mu}{d t}=-\frac{4}{3} \Theta \mu-\frac{3 \mu}{n} \frac{d n}{d t}
$$

where we have used $P_{A}=\mu / 3 n^{2}$ [see (22)], and $d / d t=U^{a} \nabla_{a}$. Of course, the analysis of the equation requires a specified density dependence of the refractive index, and therefore also a modified equation of state (for an example, see [21,22]), and will not be pursued further here.

We note the possibility to formulate (39) in terms of an effective energy-momentum tensor in certain cases, in particular for homogeneous spacetimes [23]. For example, we may define $\mu_{\text {eff }}=n^{3} \mu$, so that (40) takes the standard form for this new effective density. In general though, this is not a consistent approach [23], and can apparently only be overcome by introducing an effective geometry (see, e.g., [7]).

\section{Conclusions}

We have discussed the consequences of the different electromagnetic momentum definitions, due to Minkowski and Abraham respectively, in the context of radiation 
fluid dynamics. Starting from a kinetic description, a set of fluid equations was derived and compared for the different definitions of fluid variables. It was found that by expressing the equations in terms of certain variables, they became independent of the choice of momentum definition. Finally, from a general relativistic kinetic theory the energy-momentum conservation equation valid for a radiation gas in a refractive medium on an arbitrary spacetime, including collisional effects, was derived and discussed in a cosmological setting.

\section{Acknowledgments}

This work was supported by the Swedish Research Council through the contract No. 621-2004-3217. The author would like to thank Gert Brodin and Chris Clarkson for helpful discussions.

\section{References}

[1] D. Mihalas and B. Weibel-Mihalas, Foundation of Radiation Hydrodynamics (Dover Publications, New York, 1999).

[2] E.A. Milne, in Selected Papers on the Transfer of Radiation, ed. D.H. Menzel (Dover Publications, New York, 1966).

[3] E.G. Harris, Phys. Rev. 138 B479 (1965).

[4] G.C. Pomraning, Astrophys. J. 153321 (1968).

[5] S. Énomé, Pub. Astronom. Soc. Japan 21367 (1969).

[6] J. Bičak and P. Hadrava, Astron. Astrophys. 44389 (1975).

[7] J.L. Anderson and E.A. Spiegel, Astrophys. J. 202454 (1975).

[8] S. Kichenassamy and R.A. Krikorian, Phys. Rev. D 321866 (1985).

[9] J.D. Jackson, Classical Electrodynamics (John Wiley \& Sons, New York, 1975).

[10] W. Gordon, Ann. Phys. 7228 (1923).

[11] L.D. Landau and E.M. Lifshitz, The Classical Theory of Fields (Pergamon Press, Oxford, 1975).

[12] S.R. de Groot and L.G. Suttorp, Foundations of Electrodynamics (North-Holland, Amsterdam, 1972).

[13] I. Brevik, Phys. Rep. 52133 (1979).

[14] R.V. Jones and J.C.S. Richards, Proc. R. Soc. London, Ser. A 221480 (1954).

[15] R.V. Jones and B. Leslie, Proc. R. Soc. London, Ser. A 360347 (1978).

[16] R. Loudon, J. Mod. Opt. 49821 (2002).

[17] D.F. Nelson, Phys. Rev. A 443985 (1991).

[18] R. Loudon, L. Allen and D.F. Nelson, Phys. Rev. E 551071 (1997).

[19] A. Feigel, Phys. Rev. Lett. 92020404 (2004).

[20] G.F.R. Ellis and H. van Elst, in M. Lachieze-Rey (ed.), Theoretical and Observational Cosmology, NATO Science Series, Kluwer Academic Publishers (1998) $g r-q c / 981204604$

[21] G. Amelino-Camelia, Int. J. Mod. Phys. D 1135 (2002).

[22] S. Alexander, R. Brandenberger and J. Magueijo, Phys. Rev. D 67 081301(R) (2003).

[23] J. Triginer, W. Zimdahl and D. Pavón, Class. Quantum Grav. 13403 (1996). 